\title{
Scalaron production in contracting astrophysical objects
}

\author{
Dmitry Gorbunov ${ }^{1,2}$, Anna Tokareva ${ }^{1,3}$ \\ ${ }^{1}$ Institute for Nuclear Research of Russian Academy of Sciences, 117312 Moscow, Russia \\ ${ }^{2}$ Moscow Institute of Physics and Technology, 141700 Dolgoprudny, Russia \\ ${ }^{3}$ Faculty of Physics of Moscow State University, 119991 Moscow, Russia
}

\begin{abstract}
We study the creation of high energy SM particles in the Starobinsky model of dark energy (a variant of $F(R)$-gravity) inside the regions contracting due to the Jeans instability. In this modification of gravity the additional degree of freedom - scalaron - behaves as a particle with mass depending on matter density. So when the mass changes light scalarons could be created at a non-adiabatic stage. Later scalaron mass grows and could reach large values, even the value of $10^{13} \mathrm{GeV}$, favored by early-time inflation. Heavy scalarons decay contributing to the cosmic ray flux. We analytically calculated the number density of created particles for the case of exponential (Jeans) contraction and found it negligibly small provided the phenomenologically viable and cosmologically interesting range of model parameters. We expect similar results for a generic model of $F(R)$-gravity mimicking cosmological constant.
\end{abstract}

\section{Introduction}

Numerous observational data requires the new component in the right hand side of the Einstein equations called dark energy which cause the accelerated expansion of the Universe. The simplest and still viable candidate for the dark energy is obviously a cosmological constant. But its unnaturally small value engenders investigation of other ways to explain the observational data.

$F(R)$-gravity provides a framework for constructing models of dark energy with time dependent equation of state $p / \rho \equiv w=w(t)$, moreover, at some stage one has $w<-1$ ( see [1] for a review). Such models of modified gravity may also explain an inflationary stage of 
the early Universe providing the unified mechanism to describe both stages of accelerated expansion.

The choice of function $F(R)$ is still phenomenological to a large extent. It must be self-consistent from the theoretical point of view, explain the cosmological data and pass all solar system and astrophysical tests. The natural question is how to distinguish $F(R)$ gravity from other models of dark energy? The most straightforward way is to improve the sensitivity of overall cosmological analysis to the dark energy equation of state. However, apart of serious systematic uncertainties there are physically motivated degeneracies in the cosmological observables with respect to physical parameters. In particular, specific effects of $F(R)$-gravity at small spatial scales can be cancelled by massive (sterile) neutrino, which dynamics works against modified gravity [2].

An attractive idea of probing $F(R)$-gravity [5] is associated with possible production of high energy particles (scalarons) in space regions where matter density changes. It was claimed in Ref. [5] that the growing curvature oscillations which decay to high energy particles may lead to significant impact on the flux of ultra high energy cosmic rays. This result is rather unexpected, because high frequency oscillations (i.e. heavy particles) are produced by a slow process (the structure formation) very inefficiently. Moreover, cosmological evolution naturally gives zero initial amplitude for such oscillations, which can be associated only with the scalar mode (heavy scalaron).

In this paper we study the processes of quantum particle production in $F(R)$-gravity using the method of Bogolubov transformations. We consider $F(R)$-gravity being equivalent to normal gravity with additional scalar field (scalaron) having a complicated potential in the Einstein frame. The form of potential depends on surrounding density providing the scalaron to be a chameleon field. Scalaron is very light at densities close to the present energy density of the Universe and heavy at larger densities. It is known that the particle with time-dependent mass may be created in a quantum theory if the typical process rate is close to the mass value. Scalaron may be born being light and then its mass may grow while the object contracts. When scalaron becomes heavy it decays to high energy Standard Model particles. We consider the same $F(R)$-model as in [5], where such processes can be investigated analytically and obtain that in opposite to [5] the number density of created particles is unfortunately too small to be observed in all realistic contracting regions (astorphysical objects) in the Universe.

The paper is organized as follows. In Section 2 we describe the construction of function $F(R)$ which is appropriate both for the inflation and dark energy. In Section 3 we introduce 
the Einstein frame approach to the $F(R)$-gravity in which the additional scalar field has a mass depending on the background energy density. In Section 4 we calculate the number density of produced particles in contracting due to the Jeans instability object and discuss the particle production rate in different contracting regions of the Universe (astorphysical objects). We conclude then in Section 5.

\section{Description of the model}

The present day acceleration of the Universe expansion can be described in terms of $F(R)$ gravity by action ${ }^{1}[3]$

$$
S=\frac{M_{\mathrm{P}}^{2}}{2} \int d^{4} x \sqrt{-g} F(R)
$$

with $F(0)=0$ reflecting the disappearance of the cosmological constant in the Minkowski flat space limit.

Any viable $F(R)$ function must obey the classical and quantum stability conditions: $F^{\prime}(R)>0, F^{\prime \prime}(R)>0$. It was introduced to mimic $\Lambda$ CDM model in the late-time Universe, so in the limit of small curvatures $F(R) \approx R-2 \Lambda$ with dark energy density $\rho_{\Lambda}=\Lambda M_{P}^{2}$. Moreover, the second derivative of $F(R)$ must also be bounded from above, $F^{\prime \prime}(R)<$ const (see [4] for details) to avoid early-time singularities at $R \rightarrow \infty$. The last requirement is easily satisfied for any $F(R)$ after adding $R^{2}$-term. This term with specially selected coefficient may also provide the Starobinsky inflation in the early Universe [8].

An example of a function appropriate for the dark energy proposed by Starobinsky is [3]

$$
F(R)=R+\lambda R_{0}\left(\left(1+\frac{R^{2}}{R_{0}^{2}}\right)^{-n}-1\right) .
$$

In the regime $R \gg R_{0}$ one has $F(R) \approx R-\lambda R_{0}$, one has providing a cosmological constant. Parameter $R_{0}$ fixes a scale which corresponds to the dark energy density $\rho_{\Lambda}$ (it is valid for $\lambda \gtrsim 1):$

$$
R_{0} \equiv \frac{2}{\lambda} \frac{\rho_{\Lambda}}{M_{P}^{2}} .
$$

To avoid early-time singularity hereafter we use function $F(R)$ with the $R^{2}$-term added:

$$
F(R)=R+\lambda R_{0}\left(\left(1+\frac{R^{2}}{R_{0}^{2}}\right)^{-n}-1\right)+\frac{R^{2}}{6 M^{2}}
$$

\footnotetext{
${ }^{1}$ The metric signature is $(-+++)$.
} 
As discussed above the last term in (4) is also appropriate for the usual Starobinsky inflation [8] in the early Universe if we choose $M=3 \times 10^{13} \mathrm{GeV}$; an impact of the second term of (4) is negligible for corresponding large values of curvatures.

There is a problem (described in [4]) with the subsequent stage of scalaron oscillations. At this stage zero and even negative values of $R$ may be obtained. Then it is easy to see that later the Universe unavoidably arrives at $F^{\prime \prime}(R)<0$ leading to the quantum instability of the theory. However it is possible to construct a function $F(R)$ which provides similar late-time cosmology but does not suffer from instabilities at post-inflationary epoch. For example (here we neglect the presence of $R^{2}$-term)

$$
F^{\prime \prime}(R) \propto \frac{1}{1+\left(R / R_{0}\right)^{2 n+2}}
$$

lead to the similar to what one has with (4) results at large curvatures. In what follows we use function (4) for simplicity and being interested only in the late time Universe evolution. But we need to ensure that the evolution of $R$ does not put its value to the region of forbidden curvatures.

Starobinsky model (4) has two free parameters: $\lambda$ and $n\left(R_{0}\right.$ is fixed by (3), while $M$ is fixed to explain the early-time inflation). The stringent restriction on the value of $n$ follows from local gravity constraints on the chameleon gravity [6]. It gives $n \gtrsim 1$. And parameter

$\lambda$ is bounded only from the stability condition for the de-Sitter minimum (see for a review $[7])$. This bound mildly varies with $n$ as

$$
\lambda>f(n) \text { and } f(n) \approx n /(2 n-4 / 3)
$$

\section{Einstein frame picture: scalaron as chameleon}

$F(R)$-gravity can be considered in the Einstein frame where it describes usual Einstein gravity with an additional scalar field (scalaron) coupled to the matter fields as chameleon field [9]. The scalaron potential is

$$
V(\phi)=\frac{M_{P}^{2}}{2 F^{\prime}(R)^{2}}\left(R F^{\prime}(R)-F(R)\right),
$$

where $R=R(\phi)$ solves equation

$$
F^{\prime}(R)=e^{\frac{\sqrt{2} \phi}{\sqrt{3} M_{P}}}
$$


Through the gravity interaction scalaron couples to all the matter fields effectively described in cosmological context as ideal fluid with energy density $\rho$ and pressure $p$. This coupling modifies its potential [9]:

$$
V_{e f f}(\phi)=V(\phi)+\frac{\rho-3 p}{4} e^{-4 \bar{\phi}}
$$

The minimum $\phi_{\min }$ of $V_{\text {eff }}$ can be obtained by putting into (8) the solution $R_{\text {min }}$ of equation

$$
2 F(R)-R F^{\prime}(R)=\frac{\rho-3 p}{M_{P}^{2}} .
$$

with $\phi_{\min }$ written in terms of $R_{\min }$.

For (4) we can approximate the solution as (the greater $\lambda$, the better the accuracy)

$$
R_{\text {min }} \simeq(4+\tau) \frac{\rho_{\Lambda}}{M_{P}^{2}}, \quad \bar{\phi}_{\text {min }}=\frac{\sqrt{3} M_{P}}{\sqrt{2}} \log \left(F^{\prime}\left(R_{\text {min }}\right)\right)
$$

where

$$
\tau \equiv(\rho-3 p) / \rho_{\Lambda}
$$

The effective scalaron mass in this minimum is

$$
m_{\text {eff }}^{2}=\frac{1}{3 F^{\prime \prime}\left(R_{\text {min }}\right)}\left(1-\frac{R_{\text {min }} F^{\prime \prime}\left(R_{\text {min }}\right)}{F^{\prime}\left(R_{\text {min }}\right)}\right) \simeq \frac{1}{3 F^{\prime \prime}\left(R_{\text {min }}\right)}
$$

Thus for model (4) we obtain the scalaron mass which depends on the surrounding energy density and pressure:

$$
m_{e f f}^{2}=\frac{M^{2} m^{2}}{M^{2}+m^{2}}
$$

where

$$
m^{2}=\frac{1}{12 n(2 n+1)}\left(\frac{\lambda}{2}\right)^{2 n} \frac{\rho_{\Lambda}}{M_{P}^{2}}(4+\tau)^{2 n+2} .
$$

In a particular range of densities $m_{\text {eff }}$ strongly depends on $\tau$. Obviously (see eqs. (14), (13)) $m$ is small there, $m<M$, leading to

$4<\tau<\left(\frac{M M_{P}}{\sqrt{\rho_{\Lambda}}}\right)^{\frac{1}{n+1}}\left(\frac{2}{\lambda}\right)^{\frac{n}{n+1}}(12 n(2 n+1))^{\frac{1}{n+1}}=\left(1.4 \times 10^{55}\right)^{\frac{1}{n+1}}\left(\frac{2}{\lambda}\right)^{\frac{n}{n+1}}(12 n(2 n+1))^{\frac{1}{n+1}}$

The situation when scalaron behaves as chameleon (i.e. inequality (15) is fulfilled) can be realized in a large variety of astrophysical objects at different scales depending mostly on a choice of $n$. When mass of the particle varies with changing surrounding density such particle 
can be created if the adiabaticity condition is violated. In the next section we calculate the corresponding number of created particles.

The scalaron is unstable because it is coupled to all non-conformal fields that are presented in the matter lagrangian. If we consider the Standard model of particle physics to describe all the matter content then the scalaron presumably decays to Higgs bosons (if kinematically allowed) [10] with decay rate

$$
\Gamma=\frac{m_{e f f}^{3}}{48 \pi M_{P}^{2}}
$$

Higgs bosons decay immediately producing a flux of protons, electrons, neutrino which provide a possibility to find such events in a case of significant scalaron production.

\section{Particle production in contracting objects}

\subsection{Mathematical problem}

A particle with mass depending on the surrounding density can be produced in contracting clouds when the adiabaticity condition is violated. Consider an object contracting due to the Jeans instability with $\rho(t)=\rho_{0} e^{t / t_{J}}\left(t>0\right.$, with $\left.t_{J}=M_{P} / \sqrt{\rho_{0}}\right)$. In order to calculate the number of produced particles we use the standard approach of Bogolubov transformations described for example in a textbook [11]. According to this method we write an equation for the scalaron mode function $\varphi$ with momentum $k$ :

$$
\ddot{\varphi}+\left(k^{2}+m_{0}^{2} e^{2 \beta t}\right) \varphi=0
$$

where the definitions are

$$
m_{0} \equiv \frac{\alpha^{n+1}}{\sqrt{12 n(2 n+1)}}\left(\frac{\lambda}{2}\right)^{n} \frac{\sqrt{\rho_{\Lambda}}}{M_{P}}, \quad \alpha \equiv \frac{\rho_{0}}{\rho_{\Lambda}}, \quad \beta \equiv \frac{(n+1)}{t_{J}} .
$$

For the chosen $\rho(t)$ the contraction starts at $t=0$, so we postulate vacuum initial conditions $\varphi=1 / \sqrt{2 \omega}, \dot{\varphi}=-i \omega \varphi$ (where $\omega=\sqrt{k^{2}+m_{0}^{2} e^{2 \beta t}}$ ), at $t=-\infty$. Since $\rho=$ const for $t<0$ so such conditions may be set for any moment $t<0$, e.g., set them at $t \rightarrow(-0)$ - just before the contraction starts. The adiabaticity condition [11] ${ }^{2}$

$$
\left|\frac{\ddot{\omega}}{\omega^{3}}-\frac{3}{2}\left(\frac{\dot{\omega}}{\omega^{2}}\right)^{2}\right| \ll 1
$$

\footnotetext{
${ }^{2}$ Usually it is equivalent to the condition $\dot{\omega} / \omega^{2} \ll 1$ which means that mass of the particle must exceed the characteristic rate of the corresponding process.
} 
is violated only for $t<1 / \beta$ so after this time particles are not produced.

Equation (17) with vacuum initial conditions can be analytically solved in terms of Bessel functions. An exact form of the relevant Bogolubov coefficient is (up to an irrelevant complex phase)

$$
B=e^{\frac{\pi k}{2 \beta}} \frac{\sqrt{\pi}}{2 \sqrt{2 \beta}} \sqrt[4]{m_{0}^{2}+k^{2}}\left(\frac{i m_{0}\left(H_{i \frac{k}{\beta}+1}^{(2)}\left(\frac{m_{0}}{\beta}\right)-H_{i \frac{k}{\beta}-1}^{(2)}\left(\frac{m_{0}}{\beta}\right)\right)}{2 \sqrt{m_{0}^{2}+k^{2}}}+H_{i \frac{k}{\beta}}^{(2)}\left(\frac{m_{0}}{\beta}\right)\right)
$$

where $H_{a}^{(2)}(x)$ is the Hankel function.

Let us first discuss the case of $m_{0}<\beta$. It corresponds to the situation where the scalaron is created being light at production $(m \sim \beta)$ and a bit later, when adiabaticity condition (19) gets restored. After that its mass grows until the moment when scalaron decays to SM particles as discussed in the previous section. The number of created scalarons can be numerically obtained in this case as:

$$
n_{p}=\frac{4 \pi}{(2 \pi)^{3}} \int k^{2}|B|^{2} d k \simeq C \beta^{3}
$$

where $C=4.9 \times 10^{-4}$.

In the opposite case $m_{0}>\beta$ one expects that particle production is suppressed because massive particles cannot be created in a slow process. But numerically we obtain

$$
n_{p}=\frac{4 \pi}{(2 \pi)^{3}} \int k^{2}|B|^{2} d k=C m_{0} \beta^{2}
$$

with $C=6.23 \times 10^{-3}$. It looks strange that the larger the mass the more particles are produced. The reason is connected with the fact that the mass depends on time in a nonsmooth way in the simple mathematical model that we considered. Particles are produced mostly at the time close to $t=0$ where the mass dependence on time is not smooth. Divergent second order time derivative of $\omega$ leads to violation of adiabaticity condition (19) at $t=0$. But actually it is natural to expect that the contraction starts in a smooth way with the typical time $t_{0}>L>t_{J}$ (for causality reasons) where $L$ is a size of object. If we use such kind of smoothing we obtain an expected suppression of particle production because in this case modes evolve adiabatically.

In order to illustrate such a suppression we can consider a smooth dependence $m^{2}(t)=$ $m_{0}^{2}\left(1+e^{2 \beta t}\right)$ with $m_{0}>\beta$ and put vacuum initial conditions at $t=-\infty$. After that one can 
analytically solve the equation like (17) and obtain the Bogolubov coefficient to be (up to an irrelevant phase)

$$
B=\frac{1}{\sqrt{2 \pi q}}|\Gamma(1-i q)| e^{-\frac{\pi q}{2}}, \quad q=\frac{\sqrt{m_{0}^{2}+k^{2}}}{\beta}
$$

In the limit of large $q$ one finds $B \simeq e^{-\pi q}$. For $m_{0}>\beta$ we may write the produced number density as

$$
\begin{array}{r}
n_{p}=\frac{4 \pi}{(2 \pi)^{3}} \int k^{2}|B|^{2} d k=\frac{4 \pi \beta^{3}}{(2 \pi)^{3}} \int_{m_{0} / \beta}^{\infty}|B(q)|^{2} \sqrt{q^{2}-\left(m_{0} / \beta\right)^{2}} q d q \approx \\
\approx \frac{4 \pi \beta^{3}}{(2 \pi)^{3}} \int_{m_{0} / \beta}^{\infty} e^{-2 \pi q} \sqrt{q^{2}-\left(m_{0} / \beta\right)^{2}} q d q \sim \beta^{3} e^{-\frac{2 \pi m_{0}}{\beta}}
\end{array}
$$

We see that in a realistic model particle production is exponentially suppressed in a case of $m_{0}>\beta$. That is in accordance with the Rubakov theorem: If you do everything correctly, the result is correct. We can not calculate the exact number of produced particles in a model-independent way, because it depends on details of the Jeans instability development.

The result (21) for $m_{0}<\beta$ is still correct because particles are mostly produced not at $t \simeq 0$ but at the moment when $m \sim \beta$, mass dependence on time is smooth and our approximation works. Also it can be proved that the number of produced particles does not depend on the details of how the Jeans instability starts to evolve.

\subsection{Applications}

\subsubsection{Cosmic structure formation}

Provided the inequality (6) the condition $m_{0}<\beta$ is satisfied only for initial densities $\alpha=$

$\rho / \rho_{\Lambda}<8$ for the viable region of model parameters which corresponds to recent and ongoing structure formation processes. Using (21) we can estimate the number of created particles inside a region of typical size $L \sim 1 \mathrm{Mpc}$ :

$$
N=n_{p} L^{3}=5 \times 10^{-4} \beta^{3}(1 \mathrm{Mpc})^{3} \lesssim 10^{-12}(n+1)^{3} .
$$

We can see that only a negligible number of scalarons can be created in the structure formation process. Only the enormous value of $n$ may lead to the noticable production which we disregard. As discussed before when $m_{0}>\beta$ particle production is strongly suppressed so the earlier structure formation (star and galaxy formations) gives much smaller impact. 


\subsubsection{Star formation in our galaxy}

The density range of contracting clouds that now form stars in our Galaxy corresponds to the case $m_{0} \gg \beta$. As discussed before we can not correctly describe the particle creation process because we need to know how the Jeans instability evolves in details. But we can obtain an upper limit on the number density of created particles as $n_{p} \sim \beta^{3}$ (in reality $n_{p}$ is much smaller because of exponential suppression (25)) and calculate the corresponding flux of high energy particles:

$$
F=\frac{n_{p} L^{3}}{r^{2} t_{J}} N \sim 3(n+1)^{3} \times 10^{-78} \mathrm{~cm}^{-2} \mathrm{~s}^{-1}
$$

Here $L=c_{s} t_{J}$ is a cloud size, $c_{s} \sim \sqrt{T / m_{p}}$ is a sound speed in the gas (here we take a temperature $t=10 \mathrm{~K}$ and $m_{p}$ to be a hydrogen molecular mass), $r=10 \mathrm{kpc}$ is a characteristic distance in our galaxy and $N$ is a full number of objects that may be obtained from the known star formation rate of $3 M_{\odot}$ per year [14].

The measured flux of ultra high energy cosmic rays even at energy $10^{20} \mathrm{eV}, F \sim$ $10^{-21} \mathrm{~cm}^{-2} \mathrm{~s}^{-1}$ [13], is many orders greater than the obtained number (27). So in any case the scalaron creation has a negligible effect in astrophysics.

\subsubsection{Expanding Universe}

Expansion of the Universe obviously implies changing energy density. Scalarons are expected to be created at the moment when $m_{e f f} \sim H$ ( $H$ is a Hubble parameter), hence from dimensional analysis the number of created particles can be roughly estimated as $n_{p} \sim H^{3}$, see also [12]. One can see that there were two moments in the past of the Universe when $m_{\text {eff }}$ is close to $H$. The first moment correspond to the period just after inflation. Scalarons created at that time have decayed in the very early Universe and do not affect the present Universe. The second moment, if exists, (for large $\lambda$ scalaron mass $m_{e f f}$ is always greater than $H$ ) is very close to the present moment corresponding to the redshift $z<0.2$. So we

expect that the number density of created scalarons to be $n_{p} \sim H_{0}^{3}$ which means that there is only one particle inside the present horizon or even less.

\section{Conclusions and discussion}

We studied particle production in media with changing density which takes place in $F(R)$ gravity or other chameleon models of the dark energy. We performed a calculation of scalaron 
creation in the Einstein frame for the Starobinsky dark energy model [3]. In the case of the exponential contraction due to the Jeans instability the corresponding equation has an analytical solution. Imposing vacuum initial conditions at the moment when contraction starts we calculated the Bogolubov coefficient and the number density of created particles. In all the realistic situations the scalaron production is very inefficient. Always less than one particle inside the corresponding Jeans volume is produced. Subsequent scalaron decay contirbution to the cosmic ray flux is found to be infinitesimal. In the Starobinsky variant of $F(R)$-gravity considered here, formally, the production rate increases with parameter $n$. However the latter must be enormously huge to make the noticable effect. That implies drastic change in $F(R)$-function at the particular value of curvature $\left(R=R_{0}\right)$ which is very unnatural choice with no grounds.

We thank F. Bezrukov, A. Dolgov, K. Postnov and S. Sibiryakov for discussions. The work has been supported by Russian Science Foundation grant 14-12-01430.

\section{References}

[1] T. P. Sotiriou and V. Faraoni, Rev. Mod. Phys. 82, 451 (2010) [arXiv:0805.1726 [gr-qc]].

[2] H. Motohashi, A. A. Starobinsky and J. Yokoyama, Phys. Rev. Lett. 110, no. 12, 121302 (2013) [arXiv:1203.6828 [astro-ph.CO]].

[3] A. A. Starobinsky, JETP Lett. 86, 157 (2007) [arXiv:0706.2041 [astro-ph]].

[4] S. A. Appleby, R. A. Battye and A. A. Starobinsky, JCAP 1006, 005 (2010) [arXiv:0909.1737 [astro-ph.CO]].

[5] E. V. Arbuzova, A. D. Dolgov and L. Reverberi, Eur. Phys. J. C 72, 2247 (2012) [arXiv:1211.5011 [gr-qc]].

[6] S. Tsujikawa, Phys. Rev. D 77, 023507 (2008) [arXiv:0709.1391 [astro-ph]].

[7] A. De Felice and S. Tsujikawa, Living Rev. Rel. 13, 3 (2010) [arXiv:1002.4928 [gr-qc]].

[8] A. A. Starobinsky, Phys. Lett. B 91 (1980) 99;

[9] R. Gannouji, M. Sami and I. Thongkool, Phys. Lett. B 716, 255 (2012) [arXiv:1206.3395 [hep-th]]. 
[10] D. S. Gorbunov and A. G. Panin, Phys. Lett. B 700, 157 (2011) [arXiv:1009.2448 [hep-ph]].

[11] N. D. Birrell and P. C. W. Davies, "Quantum Fields in Curved Space," Submitted to: Cambridge Monogr.Math.Phys..

[12] Y. B. Zeldovich, Pisma Zh. Eksp. Teor. Fiz. 12 (1970) 443; Y. B. Zeldovich and A. A. Starobinsky, Sov. Phys. JETP 34 (1972) 1159 [Zh. Eksp. Teor. Fiz. 61 (1971) 2161].

[13] J. Beringer et al. [Particle Data Group Collaboration], Phys. Rev. D 86 (2012) 010001. http://pdg.lbl.gov/

[14] C. W. Allen "Astrophysical Quantities 3rd.ed.," The Athlone Press (1973) 